\title{
Aplastic Anemia in Pregnancy
}

\author{
Rathore Swati $\cdot$ Pramanick Angsumita \\ Regi Annie $\cdot$ Lionel Jessie
}

Received: 14 December 2010/ Accepted: 21 June 2011/Published online: 14 August 2013

(C) Federation of Obstetric \& Gynecological Societies of India 2013

\section{Introduction}

Although aplastic anemia was first recognized by Ehrlich in 1888, the pathogenesis of aplastic anemia has remained elusive. The prevalence of aplastic anemia in pregnancy is rare. Aplastic anemia is a subtype of anemia characterized by pancytopenia and a hypocellular bone marrow. This condition can be due to chemicals, drugs, infections, irradiation, leukemia, and inherited disorders. The treatment involves immunosuppressive therapy with antithymocyte globulin and cyclosporine and bone narrow transplantation [1]. The relationship between pregnancy and aplastic anemia remains controversial. There is universal agreement that pregnancy complicated by aplastic anemia is a serious condition [2]. The risk to the mother is mainly in the form of hemorrhage and sepsis, while the fetus may suffer from growth restriction and even intrauterine death. Hemorrhage and sepsis are responsible for more than $90 \%$ of maternal mortality [2]. Most of the fetal complications are due to maternal anemia. All along with these, maternal infections may lead to the development of chorioamnionitis and resultant preterm labor and birth [3]. In the literature, fetal thrombocytopenia, placentomegaly, and severe oligohydramnios have also been reported. We here present two

Rathore S., Assistant Professor · Pramanick A. (凹), Assistant Professor · Regi A., Professor - Lionel J., Professor Department of Obstetrics and Gynecology Unit III, Christian Medical College, Vellore 632004, India e-mail: angsumita@gmail.com cases of pregnancy complicated by aplastic anemia, which were seen within a span of 1 year at our hospital. This high incidence is because the hospital is a tertiary care referral unit with good hematology and blood bank support.

\section{Case A}

A 27-year-old primigravida, at 28 weeks of gestation, was found to have a hemoglobin level of $5.6 \mathrm{~g} / \mathrm{dL}$, during regular antenatal checkup, and was transfused $2 \mathrm{U}$ of packed cells (PC). She did not have any medical or surgical problems in the past, and her antenatal investigations had been normal. Subsequently, at 30 weeks of gestation, she developed spontaneous bruising, bleeding gums, epistaxis when a complete blood count revealed a hemoglobin level of $7.3 \mathrm{gm} / \mathrm{dL}$, white blood cell count $(\mathrm{WBC})$ of $4.0 \times 10^{9}$ \% $\mathrm{L}$ [N13L84], and platelet count of $3.8 \times 10^{9} / \mathrm{L}$ and reticulocyte at $0.2 \%$. Bone marrow biopsy was suggestive of aplastic anemia. She was advised to take cyclosporine $5 \mathrm{mg} / \mathrm{kg} /$ day (a total dose of $300 \mathrm{mg}$ ), which she took for 7 days and then defaulted. When she reported to our emergency department at 32 weeks of gestation with complaints of gum bleeding and purpuric rash, her hemoglobin was $8 \mathrm{gm} / \mathrm{dL}$, and platelets $1 \times 10^{9} / \mathrm{L}$. She was transfused $2 \mathrm{U}$ of platelet-rich concentrates (PRC). She developed severe gestational hypertension and was started on oral alpha methyl dopa $500 \mathrm{mg}$ tds. Injection dexamethasone was given for fetal lung maturation. At 33 weeks of gestation, scan showed IUGR, and with umbilical artery, Doppler study found absent end-diastolic 
flow. A multidisciplinary team consisting of obstetricians, anesthesiologists, hematologists, and neonatologists planned on offering an elective cesarean section under general anesthesia for severe IUGR and fetal compromise. Eventually, at 33 weeks and 5 days of gestation, she underwent elective cesarean section delivering a healthy preterm female baby of 1,300 $\mathrm{g}$ with Apgar scores of 9 and 9, at 1 and $5 \mathrm{~min}$, respectively. Pre-operatively, her hemoglobin was $8.4 \mathrm{~g} \%$, and she was transfused six PRCs, intraoperatively she was given six PRCs, $1 \mathrm{U}$ of PC, and $1 \mathrm{U}$ of factor-7 $(2.4 \mathrm{mg})$. There were no abnormal intraoperative findings, and surgery was uneventful. She was monitored in high dependency unit, received four PRCs and one single donor platelet unit, following which her platelet count was $10 \times 10^{9} / \mathrm{L}$, and hemoglobin was $10 \mathrm{~g} \%$.

Post-operatively, she developed fever, was started on cefotaxime and gentamicin, progressing on to imipenem and teicoplanin, which was changed to amphotericin and voriconazole because of mild infusional toxicity. During the ward stay, she was supported with $52 \mathrm{U}$ of PRCs and $9 \mathrm{U}$ of PCs. Definitive treatments of aplastic anemia like allogenic bone marrow transplant, Antithymocyte globulin (ATGAM), and Cyclosporin were offered to the patient, but she could not avail these because of financial constraints. She was discharged on request after 49 days of hospital stay and expired after 6 days of discharge. The baby, born at 33 weeks and 5 days of gestation, weighing $1,300 \mathrm{~g}$ was managed uneventfully in level 2 neonatal ICU for 22 days till it attained a weight of $1,780 \mathrm{~g}$. The baby was discharged back home in a good condition.

\section{Case B}

A 26-year-old primigravida at 10 weeks of gestation presented with bleeding per vaginum and hyperemesis. Routine blood count revealed pancytopenia with hemoglobin $8.4 \mathrm{gm} / \mathrm{dL}, \mathrm{WBC} 1.8 \times 10^{9} / \mathrm{L}$ and platelet count of $6 \times 10^{9} / \mathrm{L}$, reticulocytes $0.65 \%$, and renal function and liver function tests were normal. With a suspected aplastic anemia, she underwent bone marrow aspirate and trephine biopsy which showed a hypoplastic marrow confirming the diagnosis. Historically, there were no risk factors for aplastic anemia. Before being seen at our hospital, when she had been evaluated at 8 weeks of gestation for ecchymosis and generalized weakness, her Hemoglobin was $7.2 \mathrm{~g}$, WBC $2.6 \times 10^{9} / \mathrm{L}$, and platelets $25 \times 10^{9} / \mathrm{L}$. She had been transfused 2 U of PCs and 4 U of PRCs and referred. Clinical examination was normal except for mild pallor. Ultrasound examination revealed a normal live fetus consistent with gestational age. She and her family were explained of the potential risks to the patient and the fetus, and she chose to continue the pregnancy. She was booked as a high-risk pregnancy and managed jointly by a multidisciplinary team consisting of obstetricians and hematologists. During her antenatal period, she received five PCs and $1 \mathrm{U}$ single donor platelet. She was admitted at $38+4$ weeks of gestation with ruptured membranes, induced with prostaglandins, labor augmented with oxytocin, and she delivered normally, a healthy single live term girl baby of $2.98 \mathrm{~kg}$. There was postpartum hemorrhage of $1 \mathrm{~L}$ (atonic and traumatic) which was managed with uterotonics, prompt suturing of the second degree perineal tear by a senior professor, and vaginal packing. Intrapartum, she was transfused $2 \mathrm{U}$ of single donor platelet and two PCs, and one PC in the postnatal period. She was discharged well on the eighth postnatal day with hemoglobin of $8.9 \mathrm{~g} \%$ and platelet of $73 \times 10^{9} / \mathrm{L}$. She was followed up till 6 months during which period she gradually become transfusion independent.

\section{Discussion}

Aplastic anemia is a serious hematological disorder characterized by pancytopenia, bone marrow hypocellularity, and absence of underlying malignant or myeloproliferative disease [4, 5].

Aplastic anemia is known to increase the antenatal complications. On the literature review, the rate of preterm birth was $12.1 \%$, intrauterine death was $16.7 \%$, stillbirth was $15.1 \%$, and spontaneous miscarriage was $16.7 \%$ among pregnant women with the diagnosis of aplastic anemia [6, 7]. Although previously cited complications are commonly encountered in cases of aplastic anemia, no such complications accompanied our case. Hemorrhage at the time of delivery/abortion is another danger. Postpartum hemorrhage is an important complication among patients with the diagnosis of aplastic anemia due to decreased platelet count. Intrauterine growth retardation complicated one of our cases.

In cases of aplastic anemia, vaginal birth is preferred, and cesarean section is performed only for obstetric indications. In our case, cesarean section was performed electively, because of severe preeclampsia with IUGR and absent end diastolic flow.

In general, treatment for aplastic anemia includes withdrawal from offending drugs, supportive care, and some form of definitive therapy. Bone marrow transplantation (BMT) has been reported to be the most effective treatment, with a 5-year survival of 56-89\%. However, BMT is contraindicated during pregnancy because it requires high-doses of immunosuppressive agents or radiation therapy, which would be toxic to the fetus. Although case reports have suggested a promising result with antithymocyte immunoglobulin (ATGAM) or cyclosporine therapy during pregnancy, there is currently little 
agreement on the universal use of these therapies. The role of androgens is not clear, and androgen treatment may cause the virilization of female fetuses. The efficacy of corticosteroids or granulocyte colony-stimulating factor is also equivocal. Overall, current evidence does not favor the routine use of any drug therapy in the treatment of pregnancy-associated aplastic anemia [7]. Earlier case reports have proposed pregnancy termination as an alternative approach.

Cyclosporine $(300 \mathrm{mg} /$ day $)$ and granulocyte macrophage colony-stimulating factor $(450 \mathrm{mg}$ intravenous weekly) have been used in severe aplastic anemia after 20 weeks of pregnancy [1] though data regarding their use in pregnancy with aplastic anemia is limited. However, experience from pregnancy following organ transplant shows that cyclosporine is apparently not teratogenic. Though it is excreted in milk, fetal growth and development were found to be normal [8]. Perhaps the most important part of treatment of aplastic anemia is supportive therapy. Supportive therapy in the form of repeated blood and platelet transfusions are given to keep hemoglobin above $10.5 \mathrm{~g} / \mathrm{dL}$ and platelet count at above $20 \times 10^{9} / \mathrm{L}$.

\section{Conclusion}

Aplastic anemia is a rare complication of pregnancy. Pregnancies complicated by intrauterine growth retardation, preterm labor, stillbirth, and spontaneous abortion can be successfully followed till term if appropriate diagnostic tests and treatment modalities are employed. In our cases, in spite of good supportive care, we had a case of maternal mortality, but fetal outcome was good. Multidisciplinary team approach, intensive targeted therapy, and good financial support are necessary to achieve an optimal outcome.

\section{References}

1. Choudry VP, Gupta S, Gupta M, et al. Pregnancy associated aplastic anemia - a series of 10 cases with review of literature. Hematology. 2002;7:233-8.

2. Deka D, Malhotra N, Sinha A, et al. Pregnancy associated aplastic anemia: maternal and fetal outcome. J Obstet Gynaecol Res. 2003; 29:67-72.

3. Deka D, Banerjee N, Roy K, et al. Aplastic anemia during pregnancy: variable clinical course and outcome. Eur J Obstet Gynecol Reprod Biology. 2001;94:152-4.

4. Camitta BM, Storb R, Thomas ED. Aplastic anemia: pathogenesis, diagnosis, treatment and prognosis. N Eng J Med. 1982;306: 645-52.

5. Oosterkamp HM, Brand A, Kluin-Nelemans JC, et al. Pregnancy and severe aplastic anemia: causal relation or coincidence? $\mathrm{Br} \mathrm{J}$ Haematol. 1998;103:315-6.

6. Pavithran K, Thomas M. Pregnancy associated aplastic anemia. J Assoc Physicians India. 1996;4:273-7.

7. Kwon JY, Lee Y, Shin JC, et al. Supportive management of pregnancy-associated aplastic anemia. Int J Gynecol Obstet. 2006; 95:115-20.

8. Ohba T, Yoshimura T, Araki M, et al. Aplastic anemia in pregnancy: treatment with cyclosporine and granulocyte-colony stimulating factor. Acta Obstet Gynecol Scand. 1999;78:458-9. 